\title{
HispanismeS
}

Revue de la Société des Hispanistes Français

$18 \mid 2021$

Murs, barrières, obstacles dans les mondes hispaniques II

\section{Entre paredes: alteridades inquietantes en Tu ropa en mi armario (2010) de Bibiana Camacho}

Entre les murs: d'inquiétantes altérités dans Tu ropa en mi armario (2010) de Bibiana Camacho

Between Walls: a Disturbing Otherness in Tu ropa en mi armario (2010) from

Bibiana Camacho

\section{Lucie Valverde}

\section{OpenEdition}

Journals

\section{Edición electrónica}

URL: https://journals.openedition.org/hispanismes/14357

DOI: 10.4000/hispanismes. 14357

ISSN: 2270-0765

Editor

Société des Hispanistes Français

\section{Referencia electrónica}

Lucie Valverde, «Entre paredes: alteridades inquietantes en Tu ropa en mi armario (2010) de Bibiana

Camacho», Hispanismes [En línea], 18 | 2021, Publicado el 31 diciembre 2021, consultado el 30 enero 2022. URL: http://journals.openedition.org/hispanismes/14357 ; DOI: https://doi.org/10.4000/

hispanismes. 14357

Este documento fue generado automáticamente el 30 enero 2022.

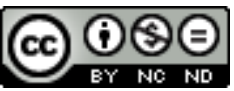

Les contenus de cette revue sont mis à disposition selon les termes de la Licence Creative Commons Attribution - Pas d'Utilisation Commerciale - Pas de Modification 4.0 International. 


\title{
Entre paredes: alteridades inquietantes en $\mathrm{Tu}$ ropa en $\mathrm{mi}$ armario (2010) de Bibiana Camacho
}

\author{
Entre les murs: d'inquiétantes altérités dans Tu ropa en mi armario (2010) de \\ Bibiana Camacho \\ Between Walls: a Disturbing Otherness in Tu ropa en mi armario (2010) from \\ Bibiana Camacho
}

Lucie Valverde

1 En el primer sentido de la palabra, las paredes sirven para cerrar o limitar un espacio. Una de sus funciones principales entonces sería distinguir el interior del exterior, definir el hogar, el espacio de lo cotidiano y de las costumbres tranquilizadoras que rigen nuestras vidas. Por lo tanto, a primera vista, estos límites no solamente nos protegen del exterior, sino que son significantes en la medida en que rechazamos fuera de estos muros lo que consideramos como otro y para protegernos de lo bárbaro: son fronteras que llevan la marca de nuestros valores ${ }^{1}$. El lugar entre paredes sería entonces el lugar de "lo mismo», el espacio privilegiado donde se puede conocer y reconocer nuestra personalidad, opuesto al exterior que sería el lugar de la alteridad.

2 En su recopilación de cuentos $\mathrm{Tu}$ ropa en mi armario', la autora mexicana Bibiana Camacho desarrolla una verdadera poética del muro, que va a cuestionar estas características que parecen consustanciales de los muros, revelando la ambigüedad de nuestros interiores. En efecto, en estos relatos, va a estallar lo cotidiano, con sus referencias reconfortantes, y los espacios entre paredes que constituían refugios donde preservar nuestra identidad misma, van a suscitar unos monstruos insospechados, invirtiendo los valores evocados anteriormente: lo interior se transformará en el lugar de una alteridad inquietante. Estos espacios, súbitamente claustrofóbicos, van a cuestionar el límite entre lo mismo y lo otro, con ocasión de unas intrusiones que revelarán lo (des)conocido. Las paredes entonces interrogarán la identidad, exponiendo 
la ambivalencia tanto de los personajes como de los hogares que en vez que resguardarlos van a volverse cárceles e incluso tumbas.

\section{Unos espacios interiores ambivalentes: una «inquietante extrañeza» en el corazón mismo de la identidad}

3 Los lugares de lo íntimo, en $T u$ ropa..., favorecen la aparición de la «inquietante extrañeza [...], esa variedad particular de lo terrorífico que se remonta a lo conocido desde hace mucho tiempo, a lo familiar desde hace mucho tiempo» según Freud, es decir lo que sería unheimlich en palabras de Schelling: «todo lo que estando destinado a permanecer en el secreto, en lo oculto, ha salido a la luz» $»^{3}$. Entre las paredes surge entonces lo monstruoso, que se traduce aquí por la inhumanidad de los comportamientos, y suscita el espanto. Utilizamos este término de «monstruo» por encarnar la anomalía, lo que se aparta de lo conocido y aceptado, subrayando una alteridad problemática, como lo explicó Jean Burgos:

Car le monstre, même familier, est gênant par cela qu'il est celui qui dévie, celui qui sort du probable et du connu, du sérieux, celui qui outrepasse la norme en tout domaine et à tous les niveaux. [...] C'est en présence de l'autre, alors, que nous met le monstre; d'un autre totalement irréductible au même et par là insituable par rapport à soi, par rapport au monde ${ }^{4}$.

4 En esta definición están todos los elementos que explican los desórdenes identitarios con que van a lidiar los protagonistas de $\mathrm{Tu}$ ropa en mi armario: este otro por su extrañeza descomunal no puede integrarse en lo idéntico, y los personajes no consiguen situarlo respecto a sí mismos. Estos monstruos amenazan el orden cuidadosamente establecido entre las paredes, hacen que allí dentro mute la realidad, la corrompen y por ende cuestionan la identidad profunda de los personajes (observaremos que existen interrelaciones entre la identidad de los muros y la de sus dueños).

5 Tendremos que recurrir a los conceptos de identidad personal y narrativa de Paul Ricœur para analizar los estragos sufridos por las interioridades (espaciales y psíquicas) de los personajes. En efecto, la pregunta a la que tienen que enfrentarse es ésta: ¿quién soy, y quiénes son ellos? La distinción entre identidad-idem e identidad-ipse ${ }^{5}$ teorizada por Ricœur entra en juego en la capacidad para contar y contarse, y veremos que los rasgos constitutivos de la identidad se ponen altamente a prueba en estos cuentos.

Interesémonos en el primer cuento de la recopilación: «Espejos». La narradora se ve obligada a visitar a los dueños del edificio donde vive, un espacio singular:

Me condujo a través de un pasillo largo y angosto, cuyos muros estaban tapizados de espejos sobrepuestos. Nuestros reflejos distorsionados se confundían y parecíamos una misma persona hecha de retazos. Dimos vuelta en un lugar donde no distinguí ninguna puerta. Llegamos a una sala amplia [...]. Las paredes, también cubiertas por espejos, parecían no delimitar el espacio ${ }^{6}$.

7 Constatamos ya que estos muros no cumplen con uno de sus requisitos, puesto que no parecen distinguir el interior del exterior. De hecho, otra incongruencia se repetirá obsesivamente a lo largo del cuento en la imposibilidad para la narradora de encontrar la puerta por la que entran y salen los demás personajes: en este espacio claustrofóbico, como por arte de magia aparecen y desaparecen seres cuya identidad no parece del todo clara, una indecisión propiciada por la multiplicación de espejos -cuya presencia 
se justifica sin embargo así en palabras de la dueña de la casa: «Así nunca olvidamos quiénes somos» ${ }^{7}$.

El reflejo parece entonces necesario para reconocer su propia identidad, y no obstante, la narradora inmediatamente se percata de que en este espacio no puede «encontrar otra cosa que no fuera [su] reflejo distorsionado». En este cuento, la intrusa es la propia narradora, por lo que la primera identidad en cuestionarse será la suya; así, el primer monstruo en exhibirse es ella misma: «La casera me escrutaba de arriba abajo, como si yo fuera un fenómeno de circo».

9 Las paredes cubiertas de espejos, así como el hecho de que los caseros nunca coinciden en la misma habitación en el mismo momento, impiden que la narradora pueda averiguar con certeza quiénes son, y en particular dudamos de que exista una pareja de caseros, sino un solo personaje que se disfrazaría. Duda entonces la narradora acerca de uno de los criterios de su identidad-idem, es decir la identidad numérica:

identité, ici, signifie unicité : le contraire est pluralité (non pas une mais deux ou plusieurs); à cette première composante de la notion d'identité correspond l'opération d'identification, entendue au sens de réidentification du même, qui fait que connaître, c'est reconnaître : la même chose deux fois, $n$ fois. [...] c'est ce qui arrive lorsque l'on parle de l'identité physique d'une personne; on n'a pas de peine à reconnaître quelqu'un qui ne fait qu'entrer et sortir, apparaître, disparaître, réapparaître ; encore le doute n'est-il pas loin, dans la mesure où l'on compare une perception présente à un souvenir récent $[. . .]^{9}$.

10 El espejo permite la sugerencia de lo doble, reforzado por la onomástica: el apellido de la pareja es Katerinov, el esposo se llama Katerin, y la esposa Katerina. Sus pocas características físicas corresponden (son pequeños y canosos) ${ }^{10}$. La descripción del esposo remite a lo idéntico: «Igualito que su mujer; la única diferencia perceptible era el cabello corto y las orejas grandes y puntiagudas» ${ }^{11}$. Sin embargo, este rasgo diferenciador se anula al final del cuento, al describir la narradora cómo Katerina «se atoraba un mechón de pelo atrás de una gran oreja puntiaguda» ${ }^{12}$.

11 La protagonista tan sólo quiere huir de este espacio -y podemos emitir la hipótesis de que el riesgo para ella sería diluirse en esta otredad física y espacial tan polifacética, y volverse uno de estos varios reflejos que sin embargo parecen uno: se llama Erika, con tan sólo dos consonantes menos que «Katerin», lo que aumenta la posibilidad inquietante de perderse a sí misma entre las numerosas identidades problemáticas encerradas entre estas paredes. Si la característica de este espacio interior se anunciaba inmediatamente como incapaz de mostrar sus límites, tampoco se puede delimitar, reconocer a los que viven allí: «El reflejo del reflejo causaba un espejismo, como si la habitación donde me encontraba no tuviera límites» ${ }^{13}$. Lo extraño parece entonces surgir entre las paredes, donde se opera una mutación de la realidad propiciada por la intrusión de lo que se considera como un monstruo, ya que Erika es, paradójicamente, el monstruo que al evidenciar una alteridad múltiple e incontenible (espacial e identitariamente) lo rompe todo al huir: «De pronto escuché un estruendo de cristales rotos que duró varios segundos. Me alejé lentamente, como si mi desorientación hubiera ocasionado el desastre e intentara huir sin levantar sospechas $»^{14}$. Todos los personajes luchan entonces por no perder la identidad -aunque mareada, Erika consigue salvarse, pero podemos suponer que el estruendo final corresponde a los espejos que se rompieron, y con ellos, la(s) identidad(es) de la(s) que eran los guardianes ${ }^{15}$. 
12 El segundo cuento de la recopilación, «La ventana», funciona también gracias a un lugar casi completamente cerrado, ya que la única abertura consiste en una ventana, que resulta problemática desde el principio. En efecto, Armanda, la narradora del cuento, no reconoce su habitación al despertar, y en particular los muros: la ventana ha cambiado de sitio ${ }^{16}$. Además, la luz exterior parece incapaz de penetrar en este lugar otra vez claustrofóbico, que se hace mortífero: «la luz agonizaba». Detrás de esta ventana no tarda en surgir una figura monstruosa (por la extrañeza que emana de ella):

Después de algunos minutos se acostumbró a la oscuridad y pudo ver, del otro lado de la ventana, a una mujer que la miraba con ojos grandes y espantados, muy erguida y completamente desnuda. No se movía; estaba como petrificada. Armanda sintió curiosidad por aquella mujer tan cercana. Observó sus pies grandes y fuertes, con uñas cortas y amarillentas. Sus pantorrillas firmes de piel reseca con vellos muy finos; las rodillas prominentes; los muslos aguados. Armanda miró las amplias caderas y el abundante vello púbico que crecía en desorden. Estiró la mano para tocar a la mujer pero, la retiró de inmediato, al ver que también la otra intentaba tocarla. [...] El cabello rizado y oscuro parecía querer escaparse; gruesos mechones se enroscaban en todas direcciones $[. . .]^{17}$.

Como aparición monstruosa, «aquella extraña» ${ }^{18}$ lleva la marca del desorden y de la otredad. Sin embargo, el lector no tarda en entender que esta monstruosidad ajena surge por la incapacidad de la narradora de identificarse a sí misma. En efecto, el cuento recurre otra vez al motivo del espejo, incomprendido por una narradora deficiente (por sufrir de Alzheimer) que cree estar frente a la ventana (tapada por las cortinas sin abrir). Aquí el elemento intruso es el espejo (nuevamente instalado por la nieta) que pone de realce la necesidad de un criterio esencial de la construcción de la identidad-idem: a la necesaria reidentificación falta la noción de continuidad ininterrumpida ${ }^{19}$.

La narradora es incapaz de reconocer su propia imagen envejecida (igualmente no consigue imaginar a «aquella mujer» más joven). Aquí, las dos vertientes de la identidad (ipse e idem) se ven afectadas:

Aussi semblable à lui-même que demeure un corps -encore n'est-ce pas le cas : il suffit de comparer entre eux les autoportraits de Rembrandt-, ce n'est pas sa mêmeté qui constitue son ipséité, mais son appartenance à quelqu'un capable de se désigner lui-même comme celui qui a son corps ${ }^{20}$.

15 Una vez vestida, Armanda sí que se reconoce a sí misma en el espejo («Antes de salir, vio su imagen en el espejo frente de [sic] la cama. Le pareció que necesitaba un peine, un poco de colorete y de rímel» ${ }^{21}$ ), pero definitivamente no integra como idéntica a sí misma la aparición otra de la mañana:

De pronto algo le hizo recordar a la mujer que había observado en la mañana, muy temprano, antes del nacimiento de la luz. Pensó que tal vez no tendría qué comer y decidió que si volvía a verla, la invitaría a desayunar y quizá le regalaría un poco de $\mathrm{ropa}^{22}$.

16 La cordura de las narradoras se ve puesta en tela de juicio en estos dos primeros cuentos, que cuestionan también el concepto de identidad: ¿de qué lado de la pared / del espejo está el otro, es decir el monstruo? Además, hasta aquí lo mismo y lo otro parecen resolverse en una sola unidad, lo que nos lleva a considerar que lo otro sería uno de los dobles de lo mismo, y que cada uno de nosotros lleva en sí mismo una parte monstruosa. Estas identidades cambiantes son problemáticas en la medida en que la identidad se caracteriza por la permanencia (opuesta a los cambios repentinos 
considerados aquí, que afectan tanto a los personajes como a los espacios en los que viven).

Los espacios de estos relatos inquietantes no presentan -a primera vista- ninguna brecha; este encierro (opuesto a la idea tranquilizadora inicial de protección obtenida gracias a las paredes) nos lleva a contemplar la existencia de un espacio exterior hasta ahí asociado a cierta normalidad, y las posibilidades de franquear estos límites que son los muros, y convertirse en intruso.

\section{Paredes que revelan lo (des)conocido e interrogan la identidad: la figura del intruso}

En los cuentos de $T u$ ropa... es fundamental la relación entre espacio e identidad. En «Espejos», por negarse a diluirse en uno de los múltiples espejismos reflejados en las paredes, Erika siente cómo el lugar se vuelve hostil y decide huir cuanto antes. En «La ventana», Armanda no reconoce los muros, y por ello no se reconoce a sí misma. En el primer caso, la intrusa (Erika) es víctima del espacio del que se siente presa; en el segundo, la intrusa (el reflejo) tan sólo subraya la magnitud del encierro de la protagonista (encerrada en su habitación y presa de su enfermedad).

19 En otras palabras, a veces lo bárbaro es otro y es posible huir de él (como en «Espejos»); en distintas ocasiones lo bárbaro «soy yo», la salvajería está dentro de mí mismo/a y afecta mi identidad propia (como en «La ventana»). Estos dos relatos muestran paredes que crean una situación de encierro; ahora bien, en otros dos cuentos, la recopilación va a extremar la sensación de reclusión al recurrir a muros que se convertirán en tumbas, mediante la intervención de intrusos que van a insinuarse en el espacio interior, provocando la muerte de sus ocupantes.

«Un ligero olor a gas», cuarto cuento de la recopilación, muestra un punto de contacto con «La ventana», por depender de un narrador deficiente: un anciano inválido, "postrado en cama» ${ }^{23} \mathrm{y}$ por ende encerrado entre unas paredes que constituyen su único horizonte. Este protagonista va dándose cuenta de que unos intrusos se han apoderado de la casa.

21 En este relato, es la permanencia de las costumbres el criterio del que depende la identidad y permite distinguir lo conocido de lo desconocido: «Puedo distinguir a cada miembro de la familia por el ruido que hace al caminar y por sus rutinas. Son tan predecibles $»^{24}$. La identidad, entonces, radica en la interacción entre los personajes y la casa. Del mismo modo, el narrador se interroga sobre su propia identidad:

Observo el techo, donde las manchas de humedad cambian constantemente, en busca de algún rasgo mío; dicen que los objetos terminan pareciéndose a quien los posee, o ¿al revés? De cualquier modo esta casa ya no es mía. Pero he pasado tanto tiempo mirando al techo que tal vez algún día aparezca mi reflejo ${ }^{25}$.

Estas reflexiones del narrador muestran que se considera a sí mismo como un intruso entre estas paredes, por no ser parte de la casa; de hecho, en este espacio se multiplican los monstruos en varias aceptaciones, y el anciano es uno de ellos: entendemos que el miedo que produce la visión de su invalidez lleva a sus familiares a mantenerlo a distancia en el último piso de la casa, de allí su sensación de «no pertenencia» ${ }^{26}$. Esta característica también hace de estos personajes unos monstruos, por su inhumanidad e insensibilidad. Pero pronto llegan otros monstruos, los ruidos entre las paredes revelan 
que lo desconocido ha irrumpido: "Alguien arrastra una silla en la cocina y luego camina cautelosamente a través de la sala. Abro los ojos de golpe. No logro identificar el ritmo de los pasos. No es nadie conocido» ${ }^{27}$.

El narrador evalúa la progresión de los intrusos (el primero, que anticipa los siguientes, es este «olor a gas» y el silencio inusual de la casa que lo acompaña) entre las paredes de la casa: la cocina, las escaleras, la habitación de la hija, el cuarto de los niños, y finalmente la propia habitación del anciano. El intruso que se ha insinuado entre las paredes pertenece entonces a otra categoría de monstruo: el del asesino repugnante, cuyo único rasgo identitario es el «aliento apestoso $»^{28}$ que llega a percibir el narrador antes de que el criminal lo ahogue con su almohada. «Un ligero olor a gas» se construye entonces en la idea de que el narrador ya no reconoce la identidad de su entorno, y más precisamente las costumbres que rigen la vida entre las paredes de la casa (paredes de las que es preso).

"La gotera», otro cuento de Bibiana Camacho, se basa en el principio opuesto: en este caso, el espacio mismo va a volverse hostil hacia la narradora, tratarla como una intrusa, antes de apresarla. La protagonista tiene que cuidar durante 3 meses el departamento de Raymundo, una ex pareja con la que había mantenido relaciones cordiales antes de perder el contacto dos años antes. Al principio, todo parece fácil:

La primera vez que fui me sentí como en casa. Conocía el espacio de memoria gracias a las fotos. El departamento, impecable, parecía la maqueta promocional de una inmobiliaria. No había nada personal a la vista: ni fotos, ni objetos, ni detalles que pudieran darme una idea del tipo de persona que para entonces podía ser Raymundo. Después de tanto tiempo de no saber de él, me parecía que ya no lo conocía y el departamento no ayudaba. Habíamos acordado los detalles mediante el correo electrónico, pero este medio nos había distanciado aún más de lo que ya estábamos ${ }^{29}$.

Se instala entonces una rutina debida a la sensación de familiaridad con el espacio, pero paulatinamente la protagonista experimenta un malestar muy particular:

El lugar era muy lindo, pero tanto orden me agobiaba y todo el tiempo que estaba dentro me sentía como una intrusa a punto de ser descubierta. Además, un ruido regular y persistente resonaba en mis sienes cada vez que visitaba el departamento; era como una gotera, pero por más que busqué alguna fuga de agua, no encontré nada $^{30}$.

Paradójicamente, la narradora no se siente autorizada en el piso, y poco a poco unos sucesos inexplicados empiezan a hacer de este departamento perfecto un lugar hostil: con frecuencia siente una presencia a sus espaldas, en una de sus visitas encuentra el papel tapiz hecho jirones (y en la visita siguiente lo vuelve a encontrar en perfecto estado, pero con un diseño distinto), un olor nauseabundo inunda el piso, etc. La identidad del lugar parece entonces problemática, ya que no muestra una permanencia necesaria -por lo menos en un referente realista. La protagonista duda entonces:

En el camino a casa pensé que podría no ser su departamento. No había nada que me lo recordara; no había visto a Raymundo ni hablado con él. El servidor me rebotaba los mensajes indicándome que la dirección de correo electrónico, con la que mantuvimos comunicación antes de su partida, ya no existía. [...] Tomé la decisión de registrar el lugar en busca de algún rastro de mi expareja. [...] Necesitaba estar segura de que me hacía cargo del departamento de alguien conocido $[. . .]^{31}$.

Ya que la legitimidad de la protagonista entre estas paredes radica en el vínculo que la une con el dueño del piso, necesita comprobar que puede reconocer, identificar sus 
huellas en ese espacio. Pero en la visita siguiente, constata que «Todo estaba vacío. No había ropa, ni papeles ni objetos personales: nada» ${ }^{32}$, y partir de entonces, «El ruido de la gotera se hizo más insistente, como si hubiera crecido» ${ }^{33}$. La narradora se convierte entonces en una verdadera intrusa, por su incapacidad de reconocer al dueño del piso el lugar donde uno vive siendo una extensión de su identidad, es el lugar de lo íntimo.

Si en el cuento "Un ligero olor a gas», es una fuga de gas la que anuncia la tragedia, mediante el olfato, aquí es el oído el que percibe la extrañeza: ruidos de gente murmurando (cuando no hay nadie), y sobre todo esta gotera que se hace cada vez más insistente, indicio de la hostilidad creciente del lugar que va a apresar a su víctima:

Me quedé dormida. Un eco constante me despertó. En el departamento inundado, varios objetos que no recordaba haber visto, flotaban en el agua que alcanzaba no menos de 30 centímetros. Me levanté y busqué la fuente de la inundación. Los grifos estaban cerrados, no había fuga visible. Las coladeras del baño y la cocina parecían tapadas. La puerta de entrada estaba atorada, ni siquiera podía mover el picaporte. Me costaba trabajo respirar [...]. Pensé que alguien derribaría la puerta en cualquier momento, la inundación tendría que ser evidente para los vecinos.

Minutos después me levanté. Las ventanas habían desaparecido de los muros. Las puertas de las demás habitaciones estaban cerradas y no pude abrirlas. Traté de gritar pidiendo auxilio, pero mi voz era sólo un susurro ${ }^{34}$.

La narradora se encuentra entonces en una situación de encierro, otra vez desaparecen las ventanas, pero en su caso ninguna puerta se abre al exterior: este departamento se hace tumba. La ambivalencia del agua, así como las variaciones que nota la protagonista, sugieren dos interpretaciones. Una consistiría en clasificar a este cuento dentro del referente fantástico - una explicación aceptable, puesto que la autora misma afirma alimentarse de miedos y pesadillas suyas. Otra nos lleva otra vez al psicoanálisis con el que habíamos abierto esta ponencia, al explicar la narradora:

Cada vez que despierto hay algo flotando que no había visto antes: un carrete de hilo, una pluma, un sombrero. El nivel del agua permanece invariable. A veces es turbia y otras es tan transparente que puedo identificar los detalles del suelo ${ }^{35}$.

Las alusiones repetidas al sueño de la protagonista, asociadas a este elemento del agua cambiante, pueden remitir a la expresión de su inconsciente -es decir de su identidad profunda. La inconstancia del lugar (opuesta a todo principio de identidad, que reposa en la noción de permanencia en el tiempo), hace del espacio entre estas paredes o el lugar metafórico del inconsciente amoroso de la protagonista (de allí las figuras fantasmales que la rodean), o un espacio a la espera de una identidad propia, reflejo de su dueño. En efecto, al comprobar que nadie vivía en este piso, la narradora afirma sentirse «en un abismo, en el vacío, en un lugar inexistente ${ }^{36}$. El que no existe, no tiene identidad, sin la cual no pueden existir los conceptos de permanencia en el tiempo y de continuidad ininterrumpida (de allí los cambios constantes e inexplicables). Este espacio sería entonces diametralmente opuesto al edificio de los espejos, que reflejaba de manera hiperbólica la identidad de su(s) dueño(s): aquí las paredes, por carecer de dueño, y por ende de identidad, se apoderan de la protagonista y le vetan toda vía de escape, obligándola a revelar su interioridad más profunda.

Para concluir, estos cuatro cuentos de los catorce que componen Tu ropa en mi armario constituyen una muestra de la verdadera poética del muro desarrollada por Bibiana Camacho (que explora muchas modalidades, de las que tan sólo hemos explorado una pequeña parte). En estos relatos, las paredes desempeñan un papel que invierte los valores asociados comúnmente a los muros: aquí, paradójicamente, el exterior es el 
lugar de lo idéntico a sí mismo, mientras que en el interior (el hogar, que alberga las acciones cotidianas y las costumbres que definen a sus dueños) surge una «inquietante extrañeza», una alteridad problemática que se manifiesta mediante figuras monstruosas. El protagonismo de las paredes permite no solamente poner en escena unos encierros turbadores, a veces sin posibilidad de escape, sino que posibilita también la irrupción de intrusos, que subrayan una dicotomía entre conocido y desconocido, entre lo que se puede identificar y reidentificar o lo que debería quedar excluido fuera, por no encajar en la identidad autorizada entre estos muros. En efecto, las paredes interrogan, reconocen y refuerzan la identidad de su dueño al reflejarla, o al contrario por no acceder a esta reidentificación lo privan de una parte de su identidad (la identidad-idem), de su amparo, y hasta pueden volverse hostiles. Las interacciones entre los muros y sus dueños tienen que ver con la afirmación de una identidad común (es decir, si podemos contestar a las preguntas ¿quién soy? y ¿es mío este lugar?), y si ésta se ve imposibilitada, la vida del protagonista está en peligro.

La monstruosidad que se exhibe entre las paredes, a salvo de las miradas ajenas, deja ver las insuficiencias de los personajes y de nuestra sociedad. Si por fuera no se nota nada extraño (una inquilina visita a sus caseros, una mujer se mira a un espejo y otra cuida el piso de un amigo que está de viaje, una familia cuida en casa de un pariente inválido), las mismas identidades personales y narrativas vistas desde dentro (desde la conciencia de sus protagonistas y entre las paredes), revelan nuestras pequeñeces o grandes tragedias cotidianas, propiciadas por otro monstruo que con frecuencia se invita en los cuentos de Bibiana Camacho y se instala a sus anchas, amparado por los muros proteicos que elevamos para protegernos del otro: el de la soledad.

\section{BIBLIOGRAFÍA}

Jean BURGoS, «Le monstre, même et autre», Jean Burgos, Léon Cellier, Jean-Pierre Collinet, Paul Mathias [et al.], Le monstre. 1, Présence du monstre : mythe et réalité, Paris, Lettres Modernes, col. Circé, Série Thématique de l'imaginaire, 1975, p. 11-24.

Bibiana CAMACHO, Tu ropa en mi armario, México, Editorial Jus, 2010.

Michel foucault, Dits et Ecrits (1954-1988), Paris, Gallimard, 1994.

Michel foucault, Les Anormaux, Cours au Collège de France. 1974-1975, Paris, Le Seuil, col. «Hautes études», 1999.

Sigmund FREUD, «L'inquiétante étrangeté», L'inquiétante étrangeté et autres essais, Paris, Gallimard, 1985, p. 213-263.

Paul RICÆUR, Soi-même comme un autre, Paris, Seuil, col. «Points», Série «Essais», 1996. 


\section{NOTAS}

1. Tomo prestada esta idea de Michel foucault, que la había aplicado en un sentido más amplio relativo a las culturas en su primer prefacio de Histoire de la folie à l'âge classique : «On pourrait faire une histoire des limites - de ces gestes obscurs, nécessairement oubliés dès qu'accomplis, par lesquels une culture rejette quelque chose qui sera pour elle l'Extérieur; et tout au long de son histoire, ce vide creusé, cet espace blanc par lequel elle s'isole, la désigne tout autant que ses valeurs.» (Michel FOUCAult, Dits et Ecrits (1954-1988), Paris, Gallimard, 1994, p. 161).

2. Bibiana CAMACHO, Tu ropa en mi armario, México, Editorial Jus, 2010. Por limitar la extensión de este artículo, nos limitaremos a la evocación de cuatro cuentos (de los catorce que forman la recopilación) para apoyar nuestra demostración: «Espejos», «La ventana», «Un ligero olor a gas» $\mathrm{y}$ «La gotera».

3. Sigmund FREUD, "L'inquiétante étrangeté», L'inquiétante étrangeté et autres essais, Paris, Gallimard, 1985, p. 222.

4. Jean BuRGos, «Le monstre, même et autre», Jean Burgos, Léon Cellier, Jean-Pierre Collinet, Paul Mathias (et al.), Le monstre. 1, Présence du monstre: mythe et réalité, Paris, Lettres Modernes, col. Circé, Série Thématique de l'imaginaire, 1975, p. 16.

5. Paul RICœUR, Soi-même comme un autre, Paris, Seuil, col. «Points», Série «Essais», 1996, p. 12-13: «La seconde intention philosophique, implicitement inscrite dans le titre du présent ouvrage par le biais du terme "même", est de dissocier deux significations majeures de l'identité [...], selon que l'on entend par identique l'équivalent de l'idem ou de l'ipse latin. L'équivocité du terme "identique" sera au cœur de nos réflexions sur l'identité personnelle et l'identité narrative, en rapport avec un caractère majeur du soi, à savoir sa temporalité. [...] L'identité, au sens d'idem, déploie elle-même une hiérarchie de significations [...] dont la permanence dans le temps constitue le degré le plus élevé, à quoi s'oppose le différent, au sens de changeant, variable. Notre thèse constante sera que l'identité au sens d'ipse n'implique aucune assertion concernant un prétendu noyau non changeant de la personnalité. [...] Dans ses acceptations variées, "même" est employé dans le cadre d'une comparaison; il a pour contraires : autre, contraire, distinct, divers, inégal, inverse. Le poids de cet usage comparatif du terme "même" m'a paru si grand que je tiendrai désormais la mêmeté pour synonyme de l'identité-idem et que je lui opposerai l'ipséité par référence à l'identité-ipse.»

6. Bibiana САМАСНО, Tu ropa en mi armario, op. cit., p. 11.

7. Ibid., p. 13.

8. Id.

9. Paul RICGUR, Soi-même comme un autre, op. cit., p. 140-141.

10. Bibiana CAMACHO, Tu ropa en mi armario, op. cit., p. 12-13.

11. Ibid., p. 13.

12. Ibid., p. 15.

13. Id.

14. Ibid., p. 16.

15. Recordemos que su función, según la dueña, es no olvidar «quiénes somos».

16. Bibiana САмАСНО, Tu ropa en mi armario, op cit., p. 17 : «Observó las paredes de su cuarto en busca de los amplios cristales que le permitían mirar al exterior. Finalmente posó la vista en el muro frente a la cama. Qué raro, pensó, la ventana estaba del lado izquierdo o del derecho, pero no enfrente.»

17. Ibid., p. 18-19.

18. Ibid., p. 19.

19. Paul RICÆUR, Soi-même comme un autre, op. cit., p. 142 : «la troisième composante de la notion d'identité, à savoir la continuité ininterrompue entre le premier et le dernier stade du développement de ce que nous tenons pour le même individu; ce critère l'emporte dans tous les 
cas où la croissance, le vieillissement, opèrent comme des facteurs de dissemblance et, par implication, de diversité numérique [...]. [...] la démonstration repose sur la mise en série ordonnée de changements faibles qui, pris un à un, menacent la ressemblance sans la détruire ; ainsi faisons-nous avec les portraits de nous-mêmes à des âges successifs de la vie ; comme on voit, le temps est ici facteur de dissemblance, d'écart, de différence.»

20. Ibid, p. 155.

21. Bibiana САMACHO, Tu ropa en mi armario, op. cit., p. 20.

22. Id.

23. Bibiana CAMACHO, Tu ropa en mi armario, op. cit., p. 27.

24. Id.

25. Ibid., p. 29.

26. Además, si seguimos la idea de Michel foucAult, el monstruo es una "figura mixta», y aquí el protagonista está vivo/muerto, inerte/animado. Para los familiares, el narrador es al mismo tiempo extraño y familiar. (Les Anormaux, Cours au Collège de France. 1974-1975, Paris, Le Seuil, col. «Hautes études», 1999, p. 59.)

27. Bibiana САMACHO, Tu ropa en mi armario, op. cit., p. 29.

28. Ibid., p. 30.

29. Ibid., p. 48.

30. Ibid., p. 48-49.

31. Ibid., p. 50-51.

32. Ibid., p. 51.

33. Id.

34. Ibid., p. 53-54.

35. Ibid., p. 54.

36. Ibid., p. 51 .

\section{RESÚMENES}

En su colección de relatos titulada Tu ropa en mi armario (2010), Bibiana Camacho despliega una verdadera poética del muro: entre las paredes (una de cuyas funciones primordiales es proteger, delimitar el hogar), la realidad se vuelve hostil, muta hasta revelar los fallos de los personajes o de nuestra sociedad. Entre estos muros, los personajes luchan por preservar su identidad y encontrar un sentido aquello que no lo tiene. Estos muros definen espacios claustrofóbicos que exploran el límite entre lo mismo y lo otro, cuestionando la identidad al sacar a la luz monstruos que alteran las normas. En esta cotidianidad de pesadilla, los espejos se convierten en guardianes de una identidad esquiva, entre la fantasía y la realidad; los intrusos revelan la profunda ambivalencia de nuestros espacios interiores, aparentes refugios que poco a poco se convierten en prisiones, incluso en tumbas.

Dans son recueil de nouvelles intitulé Tu ropa en mi armario (2010), Bibiana Camacho déploie une véritable poétique du mur : entre les murs (dont l'une des fonctions premières est de protéger, de délimiter le foyer), la réalité devient hostile, mute jusqu'à révéler les défaillances des personnages ou de notre société. Entre ces parois, les personnages luttent pour préserver leur identité et trouver un sens à ce qui n'en a pas. Ces murs définissent des espaces claustrophobes qui interrogent la limite entre le même et l'autre, remettent en question l'identité en faisant 
surgir des monstres qui altèrent les normes. Dans ce quotidien cauchemardesque, les miroirs se font gardiens d'une identité fuyante, entre fantaisie et réalité ; des intrus viennent révéler la profonde ambivalence de nos espaces intérieurs, refuges apparents qui deviennent cependant peu à peu prisons, voire tombeaux.

In her collection of short stories entitled Tu ropa en mi armario (2010), Bibiana Camacho develops an absolute poetics of the wall: between the walls (whose first function consists in protecting, delimiting the home), reality becomes hostile, mutates until it reveals the failures of the characters or of our whole society. Between these walls, the characters struggle to protect their identities or find any sense in the meaningless. These walls define claustrophobic spaces that examine the limit between the sameness and the otherness, question the concept of identity by bringing out monsters that corrupt the norms. In this nightmarish everyday life, mirrors become the keepers of an elusive identity, between fantasy and reality; some intruders appear to reveal the deep ambivalence of our indoor spaces, apparent refuges that yet gradually become prisons, or even tombstones.

\section{ÍNDICE}

Palabras claves: alteridad, identidad, muros, intrusos, encierro, desconocido Keywords: otherness, identity, walls, intruders, entrapment, unknown

Mots-clés: altérité, identité, murs, intrus, enfermement, inconnu

\section{AUTOR}

LUCIE VALVERDE

Le Mans Université - 3L.AM 